\title{
Ruptura de pseudocisto pancreático para a cavidade peritoneal: relato de caso
}

\author{
Pancreatic pseudocyst rupture to the peritonial cavity: a case report
}

Ruptura de seudoquiste pancreático para la cavidad peritoneal: relato de caso

Karla Millana Cardoso Rodrigues ${ }^{1 *}$, Diego Vinnicyus Santos Rodrigues ${ }^{2}$, Fabiola Nassar Sousa Frazão ${ }^{1}$, Orlando José dos Santos ${ }^{1}$.

\section{RESUMO}

Objetivo: Descrever a evolução e o desfecho de um caso de ruptura espontânea de pseudocisto pancreático para a cavidade peritoneal. Detalhamento do caso: Paciente do sexo masculino de 44 anos, etilista crônico, tabagista, deu entrada no pronto-socorro com queixa de dor abdominal intensa associada a vômitos e com peritonite franca ao exame físico. Exames de imagem sugeriam ruptura de pseudocisto pancreático. Submetido a laparotomia exploradora com derivação pseudocisto-gástrica, evoluiu de forma favorável e teve alta com recuperação completa do quadro. Considerações finais: Apesar de comum, a pancreatite pode trazer graves complicações. Há relato de até $5 \%$ de ruptura espontânea de pseudocistos pancreáticos e sua evolução varia de acordo com o local de erosão da coleção. A ruptura para a cavidade peritoneal pode levar a peritonite grave e necessidade de abordagem cirúrgica de urgência.

Palavras-chave: Pseudocisto Pancreático, Pancreatite, Abdômen Agudo.

\begin{abstract}
Objective: To describe the evolution and outcome of a case of spontaneous pancreatic pseudocyst rupture into the peritoneal cavity. Case detailing: A 44-year-old male patient, chronic alcoholic, smoker, was admitted to the emergency room complaining of severe abdominal pain associated with vomiting and presenting peritonitis on physical examination. Image exams suggested rupture of a pancreatic pseudocyst. He went through exploratory laparotomy with pseudocyst-gastric bypass and evolved favorably being discharged completely recovered. Final considerations: Although common, pancreatitis can lead to serious complications. There is a report of up to $5 \%$ spontaneous rupture of pancreatic pseudocysts and its evolution varies according to the erosion site of the collection. The rupture into the peritoneal cavity can lead to severe peritonitis and need for urgent surgical approach.
\end{abstract}

Key words: Pancreatic Pseudocyst, Pancreatitis, Acute Abdomen.

\section{RESUMEN}

Objetivo: Describir la evolución y el resultado de un caso de ruptura espontánea de seudoquiste pancreático en la cavidad peritoneal. Detalle del caso: Un paciente de sexo masculino de 44 años, alcohólico crónico, fumador, ingresó en la sala de emergencias que se quejaba de dolor abdominal intenso asociado con vómitos y presentaba peritonitis en el examen físico. Los exámenes de imagen sugirieron la ruptura de un seudoquiste pancreático. Fue a través de laparotomía exploratoria con seudoquiste-bypass gástrico y evolucionó favorablemente al ser completamente recuperado. Consideraciones finales: Aunque es común, la

${ }^{1}$ Universidade Federal do Maranhão (UFMA), São Luís - MA.*E-mail: karlamillana@yahoo.com.br

${ }^{2}$ Universidade Federal do Tocantins (UFT), Palmas - TO.

SUBMETIDO EM: 3/2019

ACEITO EM: 4/2019

PUBLICADO EM: 5/2019

REAS/EJCH | Vol. 11 (11) | e466 | DOI: https://doi.org/10.25248/reas.e466.2019 Página 1 de 5 
pancreatitis puede llevar a complicaciones graves. Existe un informe de hasta un $5 \%$ de ruptura espontánea de seudoquistes pancreáticos y su evolución varía según el sitio de erosión de la colección. La ruptura en la cavidad peritoneal puede conducir a una peritonitis grave y la necesidad de un abordaje quirúrgico urgente.

Palabras clave: Seudoquiste Pancreático, Pancreatitis, Abdomen Agudo.

\section{INTRODUÇÃO}

A pancreatite aguda é uma das doenças mais comuns do trato gastrointestinal. Nos Estados Unidos, representa um custo de 2,5 bilhões de dólares com mais de 275.000 admissões anuais. A incidência apresenta grande variabilidade, com especial aumento na população pediátrica. A mortalidade reduziu com o tempo, apresentando uma cifra atual de $2 \%$ (FORSMARK CE; VEGE SS; WILCOX CE, 2016). Porém ainda traz desafios em seu manejo devido a sua patogênese ainda não completamente entendida e potencial de evolução com graves complicações (BRAHA J; TENNER S, 2018). É diagnosticada naquele paciente que apresentar dois dos três seguintes critérios: dor abdominal consistente com pancreatite aguda, aumento maior que três vezes nos valores de amilase ou lípase séricos ou evidencia radiológica compatível (YADAV D; LOWENFELS AB, 2006).

As complicações de uma pancreatite aguda orbitam dentro seguinte espectro: complicações locais, sistêmicas e falência orgânica. Estas complicações locais são representadas por coleções peripancreáticas, coleções necróticas agudas, necrose pancreática delimitada e pseudocistos. (VEGE SS, 2018). A incidência dessas coleções pode chegar a 50\%, contudo a maioria delas resolve-se sem necessidade de intervenção (LERCH MM, et al., 2009; PAPADIMITRIOU N, et al., 2014).

A pancreatite crônica, comumente desenvolvida por etiologia alcoólica em 70-80\% dos casos, apresenta uma incidência anual de cinco casos em 100000 na Europa ocidental, sendo a proporção entre homens e mulheres de aproximadamente 7:1, com uma faixa etária de manifestação dentro do intervalo de 36 a 55 anos. O quadro clínico consiste de dor abdominal, que pode irradiar para o dorso, de moderada a forte intensidade, que persiste durando anos até o paciente buscar atendimento especializado. Após 10 a 15 anos de doença o paciente pode desenvolver insuficiência pancreática, com deficiência de proteínas, gorduras, esteatorreia e diabetes insulino-dependente (FRENCH JJ, CHARNLEY RM, 2016). Os pseudocistos podem ocorrer em até $25 \%$ dos pacientes com pancreatite crônica, sendo mais comum naqueles casos de etiologia alcoólica (MUJER MTP, et al., 2018).

\section{DETALHAMENTO DO CASO}

Paciente do sexo masculino de 44 anos, etilista crônico para destilados, tabagista, deu entrada no prontosocorro com queixa de dor abdominal intensa associada a vômitos. Ao exame físico, apresentava-se taquicárdico, taquipneico, sudoreico, ictérico $2+/ 4+$, hipocorado $2+/ 4+$, com dor à palpação superficial e profunda do abdome, com sinais de peritonite franca. Negava episódios prévios semelhantes, febre, hemorragia ou perda de peso. A tomografia da entrada mostrava grande quantidade de líquido livre na cavidade, além de lesão cística de limites bem definidos, contornos regulares, rechaçando anteriormente o estômago e posteriormente o pâncreas, com dimensões de $14 \times 8 \mathrm{~cm}$, além do ducto pancreático dilatado e aparente comunicação com a coleção (Figura 1).

Os exames laboratoriais não evidenciavam leucocitose, mas anemia com hemoglobina de $10 \mathrm{~g} / \mathrm{dL}$, hiperbilirrubinemia (bilirrubina total de $3,0 \mathrm{mg} / \mathrm{dL}$, com prevalência de indireta) e acentuado aumento de enzimas pancreáticas (amilase $643 \mathrm{U} / \mathrm{L}$ e lipase $1.161 \mathrm{U} / \mathrm{L}$ ).

Foi submetido à laparotomia exploradora por incisão xifopubiana, a qual teve como achados peritonite química difusa, grande quantidade de secreção achocolatada fluida e grande coleção com paredes espessas firmemente aderidas a estomago e pâncreas. Foi realizada aspiração do líquido livre, gastrostomia anterior e

REAS/EJCH | Vol. 11 (11) | e466 | DOI: https://doi.org/10.25248/reas.e466.2019 Página 2 de 5 
posterior, dando acesso ao interior do pseudocisto. Posteriormente, realizou-se a derivação da parede do pseudocisto à parede posterior do estômago por meio de cerclagem com fio absorvível de poliglactina e gastrorrafia anterior com fio não absorvível de polipropileno. Deixou-se um dreno túbulo-laminar subepático e passou-se sonda nasogástrica descompressiva e sonda nasoenteral para nutrição.

No $1^{\circ}$ dia pós-operatório (DPO), paciente evoluiu com hipotensão responsiva a volume, agitação psicomotora e episódios de hipoglicemia. O débito do dreno era de $200 \mathrm{~mL}$ de secreção sero-hemática e a sonda nasogástrica teve débito alto, de $600 \mathrm{~mL}$. Em um dos episódios de agitação, a sonda nasoenteral foi removida. A terapia antimicrobiana empregada foi com meropenem em monoterapia, mantida até o $10^{\circ}$ DPO. O paciente foi mantido em dieta oral zero e iniciado benzodiazepínico para controle de síndrome de abstinência alcoólica. $\mathrm{O}$ abdome era indolor e a ferida operatória de bom aspecto. Os exames laboratoriais de controle mostravam piora dos níveis hematimétricos, com hemoglobina de 7,5 g/dL, mas com melhora da hiperbilirrubinemia (bilirrubina total de $0,4 \mathrm{mg} / \mathrm{dL}$ ) e das enzimas pancreáticas (lípase $739 \mathrm{U} / \mathrm{L}$ e amilase 381 $\mathrm{U} / \mathrm{L}$ ). As sorologias para doenças sexualmente transmissíveis e hepatites foram negativas.

Paciente manteve o padrão de melhora clínica e laboratorial, com remoção da sonda nasogástrica e introdução de dieta oral no $4^{\circ}$ DPO, com boa aceitação de alimentação líquida restrita. O débito do dreno foi progressivamente menor até sua retirada no $7^{\circ} \mathrm{DPO}$, quando o débito se mostrou desprezível e de aspecto seroso. Foi realizada progressão de dieta até a pastosa, com boa aceitação no $7^{\circ}$ DPO. Realizou nova tomografia no $10^{\circ}$ DPO (Figura 1), que evidenciou pequena quantidade de líquido livre em pelve e discreto derrame pleural bilateral, com ausência de coleções ou abscessos.

Foi mantido meropenem até o 10ำ DPO após evidência de melhora clínica e laboratorial e ausência de sinais da presença de processos infecciosos ativos. Teve alta no $14^{\circ}$ DPO em estado geral regular com previsão de acompanhamento clínico na rede básica de saúde.

Figura 1: Tomografia de abdome realizada na admissão lesão cística de limites bem definidos, contornos regulares, rechaçando anteriormente o estomago e posteriormente o pâncreas, com dimensões de $14 \times 8 \mathrm{~cm}$.

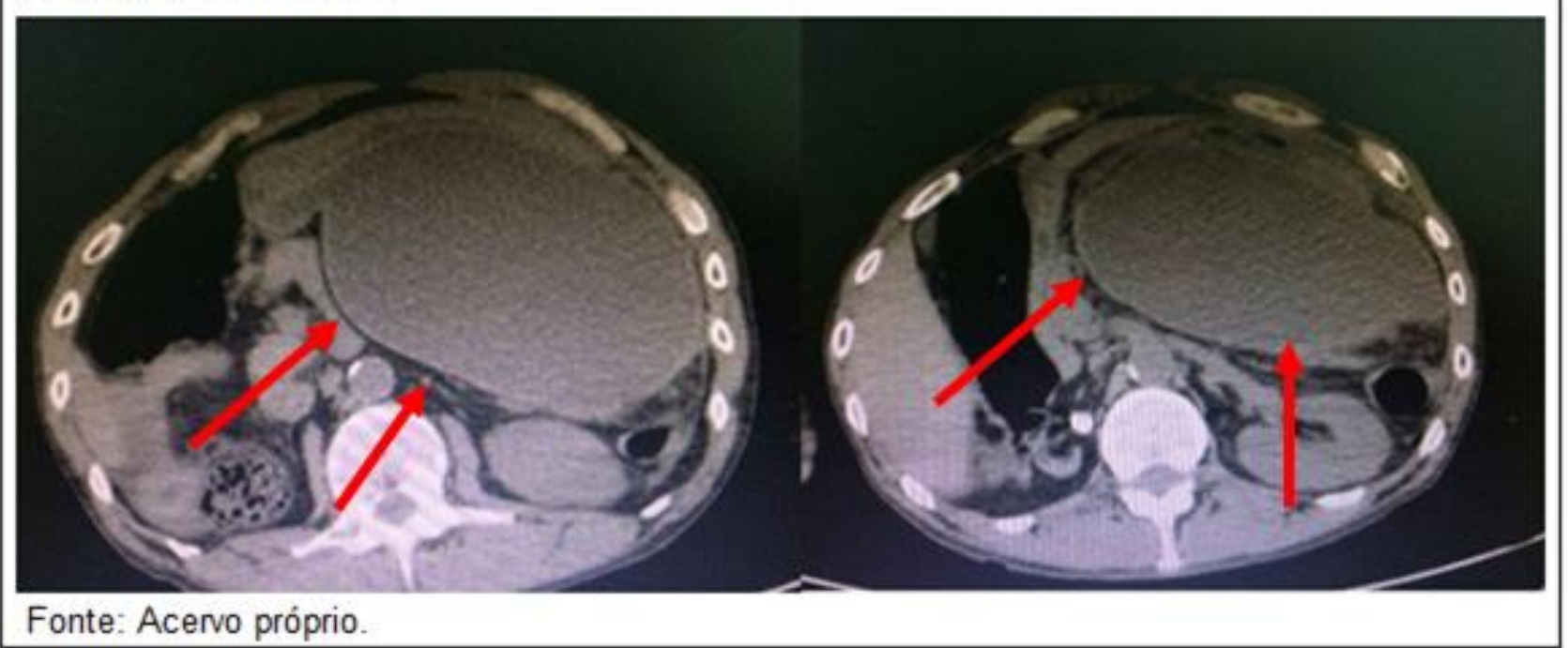

\section{DISCUSSÃO}

O pseudocisto pancreático é uma coleção peripancreática encapsulada e ocorre após quatro semanas do início de uma pancreatite aguda edematosa. Caracteriza-se pela ausência de necrose ou conteúdo sólido em seu interior e consistência fluida homogênea (VEGE SS, 2018). Os pseudocistos tendem a ocorrer em 10\%

REAS/EJCH | Vol. 11 (11) | e466 | DOI: https://doi.org/10.25248/reas.e466.2019 Página 3 de 5 
dos pacientes com pancreatite crônica, provavelmente induzidos por um episódio de pancreatite aguda ou por comprometimento do ducto pancreático (FREEDMAN SD, 2019). O caso acima, dada a história associada aos achados clínicos, consistia em um quadro de pancreatite crônica associada a uma coleção peripancreática, com características sugestivas de pseudocisto. Isto é, o paciente se encaixa na incidência supracitada e apresentou repercussões importantes, por evoluir com um quadro súbito de ruptura aliado a peritonite, o que pode ser interpretado como uma evolução atípica dos pseudocistos.

O quadro clínico do paciente com coleções peripancreáticas pode variar desde a ausência de sintomas até alterações por compressão extrínseca e a complicações sérias, como infecção e ruptura da estrutura tal qual ocorreu com o paciente acima (PAPADIMITRIOU N, et al., 2014; ROCHA R, et al., 2016; VEGE SS, 2018). O presente caso evidenciou um episódio de ruptura espontânea, que representa um evento incomum, com uma incidência reduzida tornando a descrição aqui relatada uma informação científica consistente da evolução desta enfermidade.

Há relato de até $5 \%$ de ruptura espontânea de pseudocistos pancreáticos, sendo que metade dos casos irá ocorrer pela forma de fístula para vísceras ocas, como estômago e duodeno e irão tender à resolução (PAPADIMITRIOU N, et al., 2014; ROCHA R, et al., 2016; SOMANI PO, et al., 2013). O presente relato apresentou uma evolução grave que necessitou de cuidados intensivos, ao contrário da parcela substancial que tendem à resolução. Muitos casos de pseudocistos rompidos podem ter desfechos graves a depender do sítio da ruptura, como trombose, quando houver acometimento vascular, ou como peritonite grave, quando a erosão ocorrer para a cavidade peritoneal, levando à necessidade de exploração cirúrgica de urgência assim como ocorrido no caso acima (BESSELINK M, et al., 2007; RAMACHANDRAN A, et al., 2018; ROCHA R, et al., 2016). O paciente necessitou de abordagem cirúrgica tendo em vista a apresentação súbita do quadro com peritonite, além de cuidados específicos de dieta e antibioticoterapia com meropenem. Necessitou ainda de benzodiazepínicos devido ao quadro de abstinência alcoólica, o que tornou a apresentação acima ainda mais específica.

Desde 2013, o Colégio Americano de Gastroenterologia recomenda apenas realizar a drenagem em caso de sintomas ou complicações. O manejo eletivo compõe os procedimentos menos invasivos, como drenagem externa percutânea e drenagem interna por endoscopia, ou as alternativas cirúrgicas, como cistogastroanastomose, cistojejunoanastomose e cistoduodenoanastomose. A escolha pela técnica depende das condições clínicas e das relações anatômicas do pseudocisto (MARTINÉZ-ORDAZ JL, et al., 2016). No paciente do caso, optou-se pela alternativa cirúrgica devido à presença de peritonite difusa com necessidade de toalete da cavidade. A derivação cistogástrica foi eleita pela intimidade da coleção com a parede posterior do estômago.

\section{CONSIDERAÇÕES FINAIS}

Apesar de comum, a pancreatite pode trazer graves complicações. Há relato de até $5 \%$ de ruptura espontânea de pseudocistos pancreáticos e sua evolução varia de acordo com o local de erosão da coleção. A ruptura para a cavidade peritoneal pode levar a peritonite grave e necessidade de abordagem cirúrgica de urgência.

\section{REFERÊNCIAS}

1. BESSELINK M, et al. Rupture of infected peripancreatic necrosis to the peritoneal cavity with fatal outcome. Pancreas, 2007; 34(4): 477-479.

2. BRAHA J, TENNER S. Fluid Collections and Pseudocysts as a Complication of Acute Pancreatitis. Gastrointest Endosc Clin N Am, 2018; 28(2):123-130.

3. FORSMARK CE, VEGE SS, WILCOX CE. Acute Pancreatitis. N Engl J Med, 2016; 375: 1972-1981. 
4. FREEDMAN, SD. Overview of the complications of chronic pancreatitis. 2019. In: UpToDate. Disponível em: https://www.uptodate.com/contents/overview-of-the-complications-of-chronic-pancreatitis. Acesso em: 21 maio 2019.

5. FRENCH JJ, CHARNLEY RM. Chronic Pancreatitis. Surgery-Oxford International Edition, 2016 ; 34 (6): 301 306.

6. LERCH MM, et al. Pancreatic pseudocysts: observation, endoscopic drainage, or resection? Deutsches Arzteblatt, 2009; 106(38): 614-621.

7. MARTINÉZ-ORDAZ JL, et al. Surgical treatment of pancreatic pseudocysts. Cirugía e cirujanos, 2016; 84(4): 288-292.

8. MUJER MTP, et al. Spontaneous rupture of a pancreatic pseudocyst. BMJ Case Rep, 2018.

9. PAPADIMITRIOU N, et al. Spontaneous intra-gastric walled-off pancreatic necrosis rupture. JOP, 2014; 15: 628629.

10. RAMACHANDRAN A, et al. Pancreatic Walled-Off Necrosis Eroding into the Inferior Vena Cava. Current Problems in Diagnostic Radiology, 2018. https://doi.org/10.1067/j.cpradiol.2018.01.007

11. ROCHA R, et al. Spontaneous Rupture of Pancreatic Pseudocyst: Report of Two Cases. Case Reports in Surgery, 2016; 2016: 1-3.

12. SOMANI PO, et al. Uncomplicated spontaneous rupture of pancreatic pseudocyst into stomach: A case report. World J Gastrointest Endosc 2013; 5: 461-464.

13. VEGE SS. Clinical manifestations and diagnosis of acute pancreatitis. 2018. In: UpToDate. Disponível em: https://www.uptodate.com/contents/clinical-manifestations-and-diagnosis-of-acute-pancreatitis. Acesso em: 20 set. 2018.

14. YADAV D, LOWENFELS AB. Trends in the epidemiology of the first attack of acute pancreatitis: a systemic review. Pancreas, 2006; 33: 323-330. 